\title{
Inactivation of uptake hydrogenase leads to enhanced and sustained hydrogen production with high nitrogenase activity under high light exposure in the cyanobacterium Anabaena siamensis TISTR 8012
}

\author{
Wanthanee Khetkorn ${ }^{1}$, Peter Lindblad ${ }^{2^{*}}$ and Aran Incharoensakdi ${ }^{1 *}$
}

\begin{abstract}
Background: Biohydrogen from cyanobacteria has attracted public interest due to its potential as a renewable energy carrier produced from solar energy and water. Anabaena siamensis TISTR 8012, a novel strain isolated from rice paddy field in Thailand, has been identified as a promising cyanobacterial strain for use as a high-yield hydrogen producer attributed to the activities of two enzymes, nitrogenase and bidirectional hydrogenase. One main obstacle for high hydrogen production by A. siamensis is a light-driven hydrogen consumption catalyzed by the uptake hydrogenase. To overcome this and in order to enhance the potential for nitrogenase based hydrogen production, we engineered a hydrogen uptake deficient strain by interrupting hupS encoding the small subunit of the uptake hydrogenase.

Results: An engineered strain lacking a functional uptake hydrogenase (AhupS) produced about 4-folds more hydrogen than the wild type strain. Moreover, the $\Delta$ hupS strain showed long term, sustained hydrogen production under light exposure with 2-3 folds higher nitrogenase activity compared to the wild type. In addition, HupS inactivation had no major effects on cell growth and heterocyst differentiation. Gene expression analysis using RT-PCR indicates that electrons and ATP molecules required for hydrogen production in the ShupS strain may be obtained from the electron transport chain associated with the photosynthetic oxidation of water in the vegetative cells. The $\Delta$ hupS strain was found to compete well with the wild type up to $50 \mathrm{~h}$ in a mixed culture, thereafter the wild type started to grow on the relative expense of the $\Delta$ hupS strain.
\end{abstract}

Conclusions: Inactivation of hupS is an effective strategy for improving biohydrogen production, in rates and specifically in total yield, in nitrogen-fixing cultures of the cyanobacterium Anabaena siamensis TISTR 8012.

Keywords: Anabaena siamensis, Heterocyst differentiation, HupS inactivation, Hydrogen production, Nitrogenase activity, Uptake hydrogenase

\section{Introduction}

The $\mathrm{N}_{2}$-fixing cyanobacterium Anabaena siamensis TISTR 8012, a novel strain isolated from rice paddy field in Thailand has been reported to have a high potential

\footnotetext{
* Correspondence: Peter.Lindblad@kemi.uu.se; aran.i@chula.ac.th ${ }^{2}$ Photochemistry and Molecular Science, Department of Chemistry Ångström Laboratory, Uppsala University, Box 523, SE-75120, Uppsala, Sweden 'Laboratory of Cyanobacterial Biotechnology, Department of Biochemistry, Faculty of Science, Chulalongkorn University, Bangkok 10330, Thailand
}

for hydrogen production with the ability to utilize sugars as substrate to produce hydrogen [1]. In Anabaena, there may be three enzymes directly involved in hydrogen metabolism. 1) Nitrogenase, a multiprotein enzyme complex consisting of two proteins, dinitrogenase (MoFe protein), encoded by nifD and nifK, and the dinitrogenase reductase (Fe protein), encoded by nifH. This enzyme catalyzes the reduction of atmospheric $\mathrm{N}_{2}$ to ammonia as well as the reduction of proton $\left(\mathrm{H}^{+}\right)$to hydrogen $[2,3]$. In the 
absence of the substrate $\mathrm{N}_{2}$, nitrogenase may exclusively catalyze hydrogen production. 2) Uptake hydrogenase, a heterodimeric enzyme with at least two subunits, HupS (small subunit) and HupL (large subunit). The large subunit, encoded by hupL, contains the active site, consisting of four conserved cysteine residues that are involved in the coordination of the metallic NiFe at center of the active site. The small subunit, encoded by hupS, contains three FeS clusters which have a function in transferring electrons from active site of HupL to the electron transport chain. The physiological function of the uptake hydrogenase is recycling of hydrogen produced by nitrogenase [2-5]. 3) Bidirectional hydrogenase, a heteropentameric, $\mathrm{NAD}^{+}$-reducing enzyme, encoded by hoxEFUYH. It consists of two protein complexes; hydrogenase (HoxY and $\mathrm{HoxH}$ ) and a diaphorase unit (HoxE, HoxF and HoxU). The bidirectional hydrogenase is commonly found, though not universal, in both $\mathrm{N}_{2}$-fixing and non- $\mathrm{N}_{2}$-fixing cyanobacteria and catalyzes both consumption and production of molecular hydrogen $[2,3,6]$.

In $A$. siamensis, an enhanced hydrogen production is mainly achieved through the nitrogenase enzyme [7]. However, the net hydrogen yield is lost due to the activity of the uptake hydrogenase. To overcome this, we engineered a hydrogen uptake deficient strain by interrupting hupS with an antibiotic resistance cassette. Previous studies have reported that $\mathrm{N}_{2}$-fixing cyanobacteria such as Nostoc punctiforme, Anabaena sp. strain PCC 7120, Anabaena variabilis and Nostoc sp. strain PCC 7942 with inactivated uptake hydrogenases show an ability to produce hydrogen at higher rate when compared to their corresponding wild type strains [8-12]. Interestingly, previous reports mainly focused on HupL inactivation since the active site of uptake hydrogenase is located in the large subunit. Therefore, we focused on HupS in A. siamensis TISTR 8012. The structural hupS and hupL genes of A. siamensis have been identified and sequenced [13]. hupS is located upstream of hupL and the predicted gene products for hupS and hupL consist of 320 and 531 amino acids, respectively. Their deduced amino acid sequences show higher than $90 \%$ and $80 \%$ similarity for HupS and HupL, respectively when compared to other cyanobacteria [13]. RT-PCR analysis revealed that hupS and hupL were co-transcribed with an enhanced transcription when the cells were grown under $\mathrm{N}_{2}$-fixing condition [13]. HupS and HupL of A. siamensis and other cyanobacteria need to go through a maturation process to become a fully functional enzyme [14].

Thus, in the present study we engineered a strain lacking a functional uptake hydrogenase $(\Delta h u p S)$ with the aim to enhance hydrogen production in $A$. siamensis TISTR 8012. In addition, the nitrogenase activity and transcript levels of genes involved in hydrogen metabolism and photosynthetic pathways in the $\Delta$ hupS strain were investigated. As expected, the $\Delta$ hupS strain was more efficient in hydrogen production under long term of light exposure than the wild type strain and the production could be prolonged for more than $72 \mathrm{~h}$ under light conditions.

\section{Results and discussion}

The confirmation on a complete segregation of a $\Delta$ hupS strain of Anabaena siamensis

After transformation of recombinant plasmid (pRLhupSNm) into cells of Anabaena siamensis TISTR 8012 (Figure 1), recombinant colonies were selected on BG11 plate containing $25 \mathrm{ug} \mathrm{mL} \mathrm{L}^{-1}$ of neomycin and transferred to BG11 broth containing antibiotic at the same concentration before analyzing for complete segregation using colony PCRs. To ensure complete segregation of $\Delta$ hupS cells, colony PCRs were performed by using a primer pair specific to hupS as shown in Figure 2A. The results show that PCR products obtained from different recombinant colonies after two weeks did not show complete segregation whereas the completely segregated recombinant strains were found after 4 weeks of cultivation (Figure 2B). A completely segregated recombinant strain was selected for further analysis.

\section{Effect of hupS inactivation on hydrogen production, growth rate and heterocyst differentiation}

The physiological characterization of the $\Delta h u p S$ strain of A. siamensis TISTR 8012 was investigated by comparision with the corresponding wild type strain. A. siamensis TISTR 8012 wild type and $\Delta$ hupS strain were grown in media with combined N-source (BG11) and without $\mathrm{N}$-source $\left(\mathrm{BG} 11_{0}\right)$. Samples were taken to measure the optical density of cell culture every three days of cultivation. The results showed that even though the growth rate of the wild type and $\Delta$ hupS strains had a similar pattern in both media the $\Delta$ hupS strain grew slightly slower than the wild type strain (Figure 3A, B), suggesting that HupS inactivation had only minor effects on cell growth. This is in agreement with earlier observations using other filamentous cyanobacterial strains [8-12]. Moreover, under $\mathrm{N}_{2}$-fixing condition there was no discernible difference in physiological morphology between the $\Delta h u p S$ and wild type strains when observed under the Scanning Electron Microscope (SEM) (Figure 3C,D) and the heterocyst frequency in the $\Delta h u p S$ filaments gradually increased with time in a similar manner to that in the wild type (data not shown).

Furthermore, the transcription levels of key genes involved in heterocyst differentiation, $n t c A$ and hetR, were examined under $\mathrm{N}_{2}$-fixing conditions by RT-PCR. The transcription factor NtcA encoded by $n t c A$, is a key transcriptional factor required for the activation of many genes involved in nitrogen and carbon metabolism $[15,16]$. 


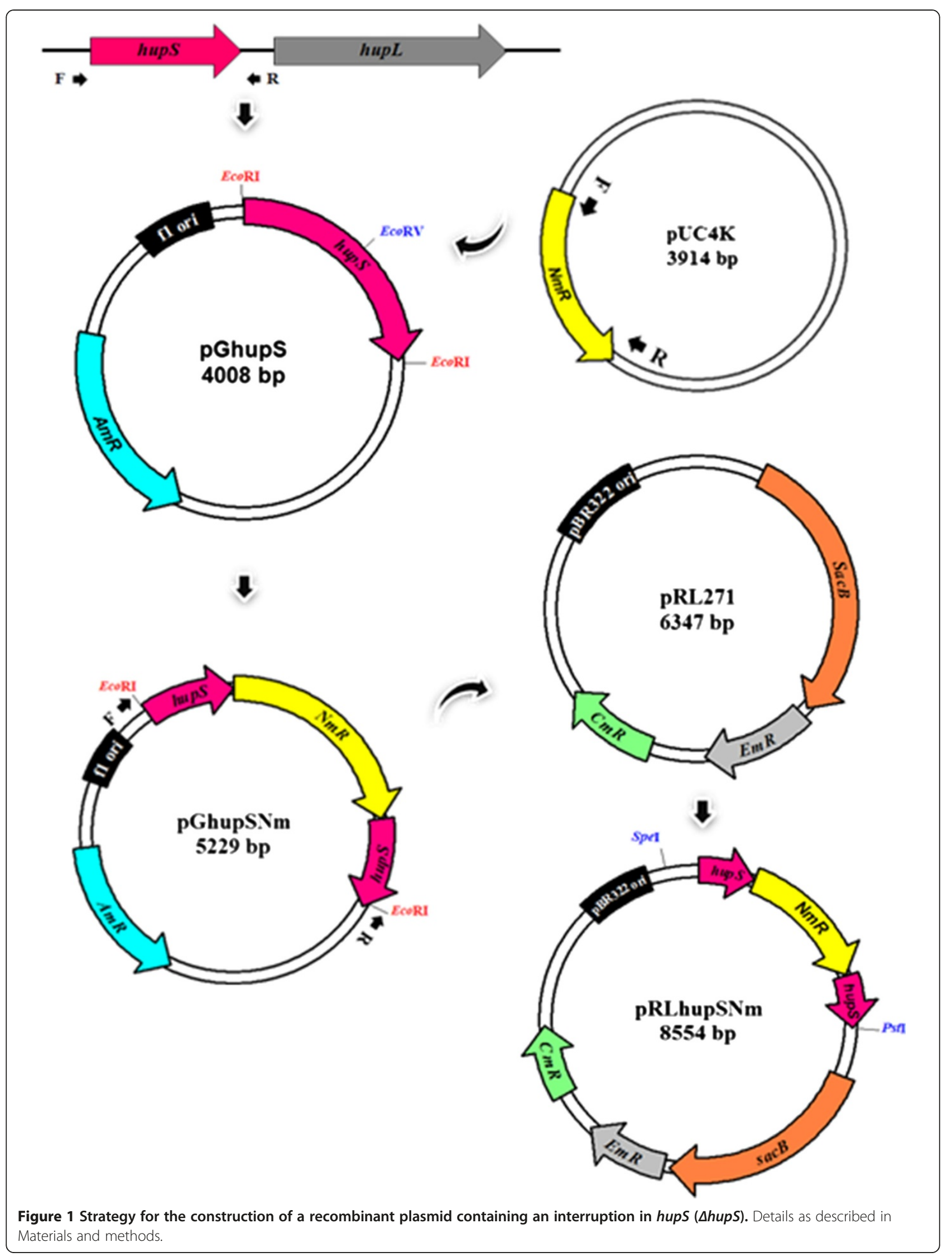



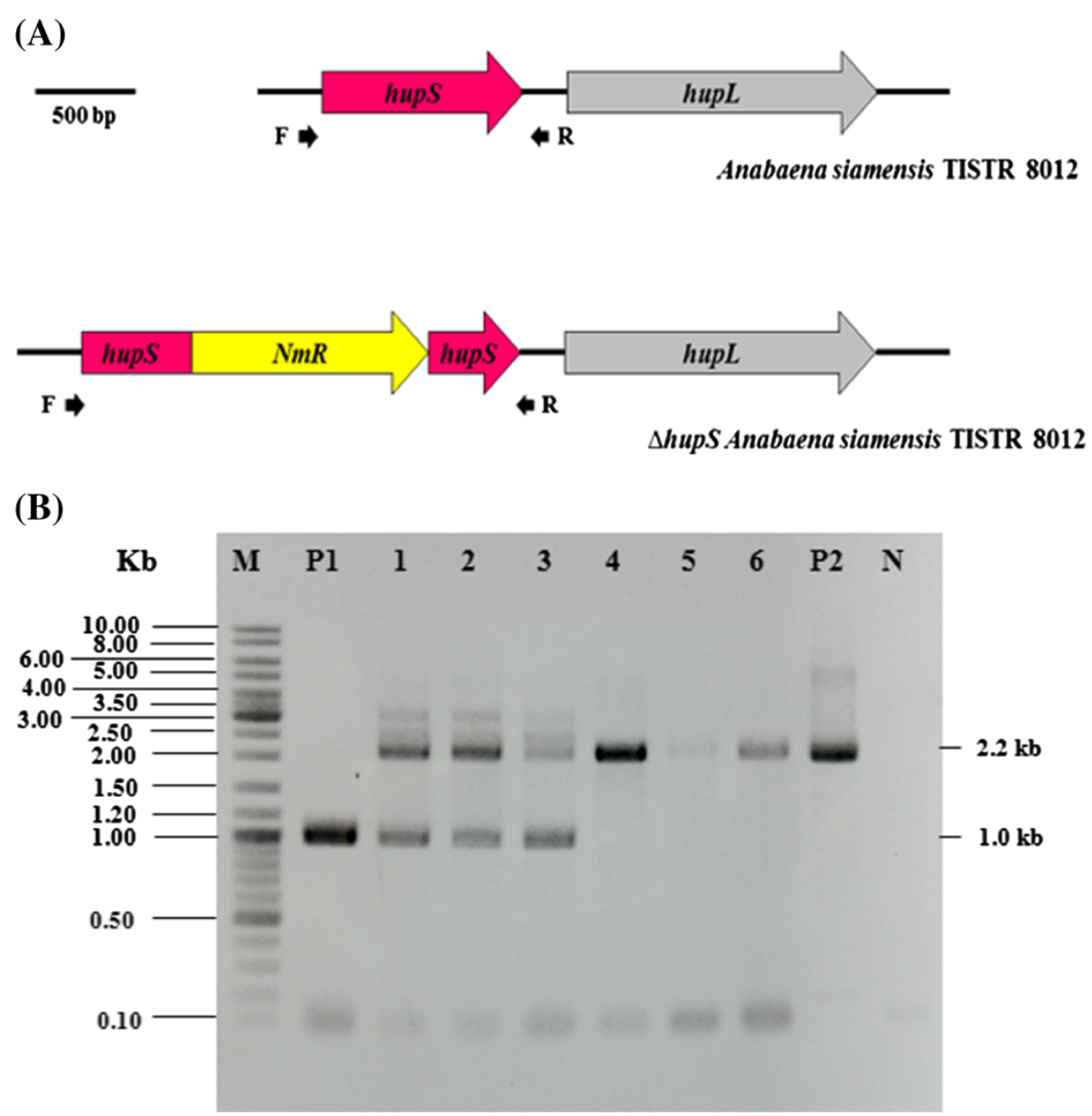

Figure 2 Engineering of a LhupS strain of Anabaena siamensis TISTR 8012. (A) Physical map of hupSL in wild type A. siamensis TISTR 8012 and an engineered strain lacking a functional uptake hydrogenase ( $($ hupS) created by interrupting hupS with neomycin antibiotic resistant cassette. (B) Confirmation of complete segregation of $\Delta$ hupS was performed by using colony PCRs and analyzed by $0.8 \%$ agarose gel electrophoresis. Primer pairs specific to hupS was used; Lane M: GeneRuler ${ }^{\mathrm{TM}}$ DNA ladder (Fermentas), Lane P1: Positive control (PCR product using genomic DNA of wild type as template), Lanes 1-3: PCR products of recombinant colonies cultured in BG11 medium in which the addition of antibiotic for 2 weeks did not show complete segregation, Lanes 4-6: PCR products of recombinant colonies cultured in BG11medium in which the addition of antibiotic for 4 weeks did show complete segregation, Lane P2: Positive control (PCR product using pRLhupSNm plasmid as template), Lane $\mathrm{N}$ : Negative control using $\mathrm{H}_{2} \mathrm{O}$ as template.

In addition, NtcA is also required for the development and function of mature heterocysts. HetR, a serine-type protease encoded by hetR, is expressed early during heterocyst differentiation and is crucial to the differentiation process [17]. Mutation in the hetR inhibits early steps in the formation of heterocysts while over-expression of hetR gives rise to multiple heterocysts [18]. Both NtcA and HetR are auto-regulated $[19,20]$. The experiments revealed no discernible differences in either $n t c A$ or het $R$ transcript levels between $\Delta$ hupS and wild type strains after $72 \mathrm{~h}$ cultivation (Figure 4A). This suggests that HupS is not essential for heterocyst differentiation.

When analyzing hydrogen production, the wild type and hupS strains of A. siamensis TISTR 8012 were grown under $\mathrm{N}_{2}$-fixing conditions (BG11 $1_{0}$ medium) for
12, 24, 48, and $72 \mathrm{~h}$, respectively. Hydrogen production was then determined under continuous illumination of $40 \mu \mathrm{Em}^{-2} \mathrm{~s}^{-1}$ and anaerobic condition for $12 \mathrm{~h}$. Interestingly, the $\triangle$ hupS strain produced hydrogen at a significantly higher rate than that of the wild type (Figure 4B). The maximum hydrogen production rate of the $\Delta h u p S$ strain was $29.7 \mu \mathrm{mol} \mathrm{H}_{2} \mathrm{mg} \operatorname{chl} a^{-1} \mathrm{~h}^{-1}$ when grown in $\mathrm{BG} 1_{0}$ medium for $72 \mathrm{~h}$, which is almost 4-folds higher than that observed in the wild type under normal growth condition. These results demonstrate that inactivation of hupS is an effective strategy for improving cyanobacterial photobiological hydrogen production in A. siamensis TISTR 8012. Similar observations have earlier been made in other filamentous cyanobacterial strains [8-12]. 

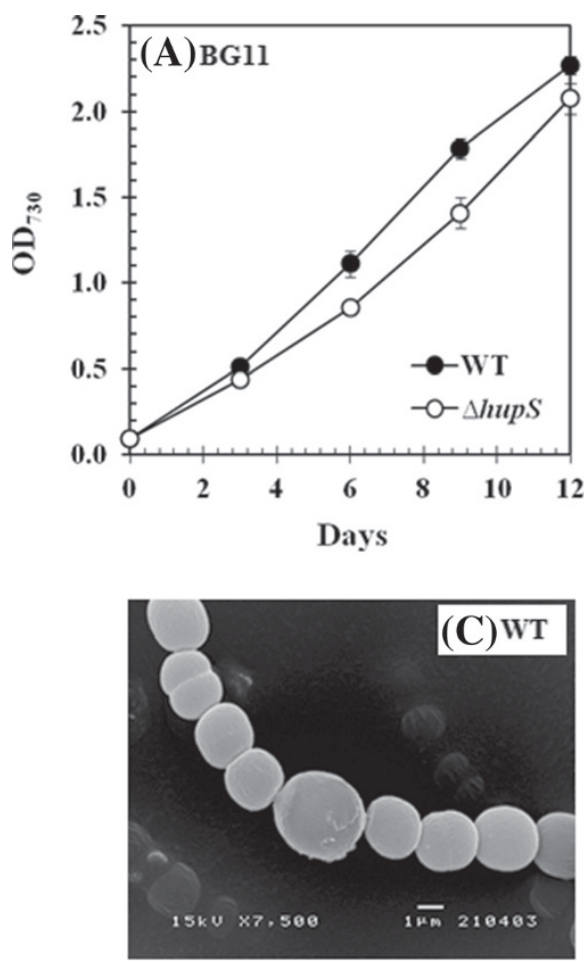
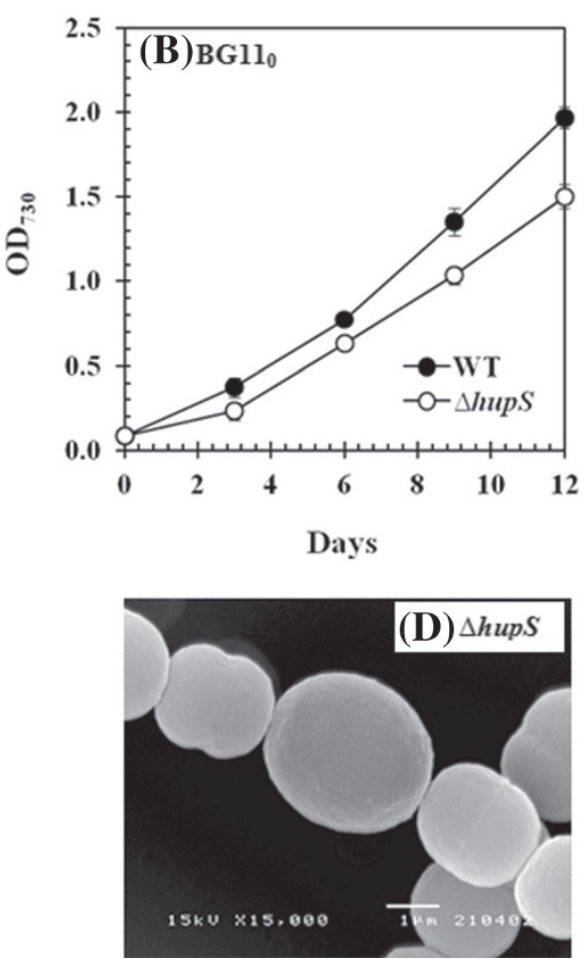

Figure 3 Characterization of wild type and $\Delta$ hupS strains of Anabaena siamensis TISTR 8012. Comparison of growth rate between wild type and the $\Delta$ hupS strains when cells were grown in either BG11 (A) or BG11 (B) medium, means \pm S.D. ( $n=3$ ). Error bars are included in the graphs, some may be smaller than the symbols used. The morphology of wild type (C) and $\Delta$ hupS strain (D) of A. siamensis TISTR 8012 cells observed under Scanning Electron Microscope (SEM) grown without the addition of N-source (BG110 medium).

\section{Sustained hydrogen production and enhanced nitrogenase activity under long term of light exposure in the $\Delta$ hupS strain}

We have previously reported that hydrogen production of wild type cells of $A$. siamensis TISTR 8012 decreased when the cells during the production phase were subject to long duration of high light intensity exposure $\left(200 \mu \mathrm{E} \mathrm{m} \mathrm{m}^{-2} \mathrm{~s}^{-1}\right)$ with the consequence of high uptake hydrogenase activity [1]. Therefore, the $\Delta h u p S$ strain was grown under $\mathrm{N}_{2}$-fixing condition followed by measuring light dependent hydrogen production for various times up to $96 \mathrm{~h}$. Interestingly, the $\Delta$ hupS strain showed much higher hydrogen production under long term light exposure than the wild type strain (Figure 5A). The hydrogen production rate in the $\Delta$ hupS strain continued to increase even after exposure to light longer than $12 \mathrm{~h}$ and the production could be prolonged up to $96 \mathrm{~h}$ under high light conditions. The nitrogenase activity of the $\Delta$ hupS strain was also investigated under the same conditions. The $\Delta$ hupS strain showed increased nitrogenase activity upon incubation up to $96 \mathrm{~h}$ with maximum activity detected after $24 \mathrm{~h}$ (Figure 5B). In contrast, the wild type strain had about 2-3 fold lower nitrogenase activity than the $\Delta$ hupS strain. A previous study in $A$. variabilis mutant strain AVM13 in which hupL was interrupted showed no difference of nitrogenase activity when compared to wild type strain. Moreover, the higher hydrogen production by the AVM13 strain under $\mathrm{N}_{2}$-fixing conditions was not sustainable and a dramatically decreased hydrogen production was detected after $30 \mathrm{~h}$ incubation [8]. Our results demonstrate that the $\Delta$ hupS strain of $A$. siamensis TISTR 8012 has a high potential for hydrogen production and an ability for long term, sustained hydrogen production under light exposure.

\section{Transcription levels of genes related to hydrogen} metabolism and photosynthesis in wild type and $\Delta$ hupS The $\Delta$ hupS strain was examined for the relative transcript levels of genes encoding proteins involved in hydrogen metabolism and the photosynthesis pathway in order to provide important information for the regulation of hydrogen metabolism as affected by hupS inactivation. Significantly enhanced nifD but not hoxH transcript levels under $\mathrm{N}_{2}$-fixing conditions were observed in the $\Delta$ hupS strain (Figure 6A). This indicates that the increased hydrogen production in the $\Delta$ hupS strain may largely be due to higher nitrogenase activity with little or no contribution from the bidirectional hydrogenase. However, since the uptake hydrogenase which is one of the enzymes to supply 


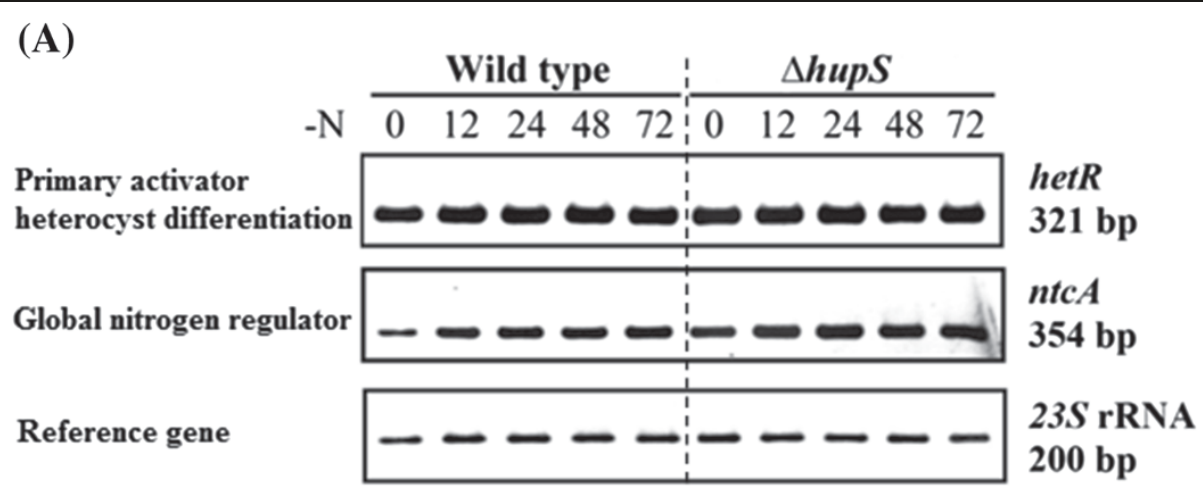

(B)

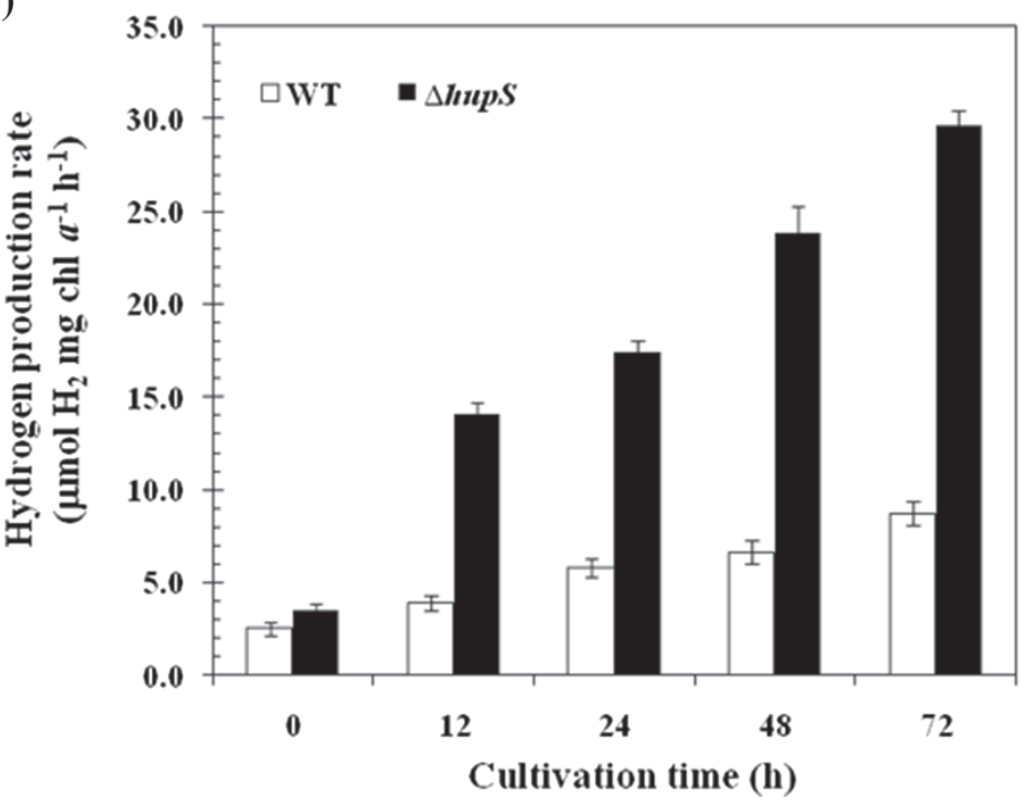

Figure 4 Effect of HupS inactivation on genes transcription and hydrogen production. Comparison of transcription levels of hetR and ntcA genes (A) and hydrogen production (B) between wild type and ShupS strains of Anabaena siamensis TISTR 8012 when grown in BG110 medium for various times at $40 \mu \mathrm{Em}^{-2} \mathrm{~s}^{-1}$. Hydrogen production rate was determined under continuous illumination of $40 \mu \mathrm{Em} \mathrm{m}^{-2} \mathrm{~s}^{-1}$ and anaerobic condition for 12 h. Means \pm S.D. $(n=3)$.

electrons to the nitrogen fixation process is inactivated, the nitrogenase is likely to receive ATP and reducing equivalents from other pathways. The transcript levels of $p s b A$, encoding the D1 protein of photosystem II, and $f d x H$, encoding a heterocystspecific ferredoxin mediating electron transport to the nitrogenase in heterocysts, were significantly upregulated in the $\Delta$ hupS strain (Figure 6B).

This may suggest that electrons and ATP needed for hydrogen production in the $\Delta$ hupS strain of A. siamensis TISTR 8012 can be obtained from the electron transport chain associated with the photosynthetic oxidation of water of photosystem II in the vegetative cells. In addition, there was no change observed in the transcription level of the psaA encoding the core protein of photosystem I (Figure 6B). It should be noted that the source of electron transfer to nitrogenase could arise from not only vegetative cells but also from within the heterocysts. Previously, proteins involved in the oxidative pentose phosphate pathway have been reported to be more abundant in heterocysts of a hydrogen uptake deficient strain of Nostoc punctiforme [21].

The inactivation of HupS of A. siamensis TISTR 8012 $(\triangle h u p S)$ resulted in a significant up-regulation of $\operatorname{cox} A$ encoding the cytochrome $\mathrm{c}$ oxidase subunit I which is present in vegetative cells only [22]. The increase of CoxA activity would lower the level of $\mathrm{O}_{2}$ in vegetative cells resulting in less inhibition of bidirectional hydrogenase, leading to enhanced hydrogen production. Nevertheless, to further explore the effect of HupS inactivation on cell metabolism, the global protein expression level should be investigated. 

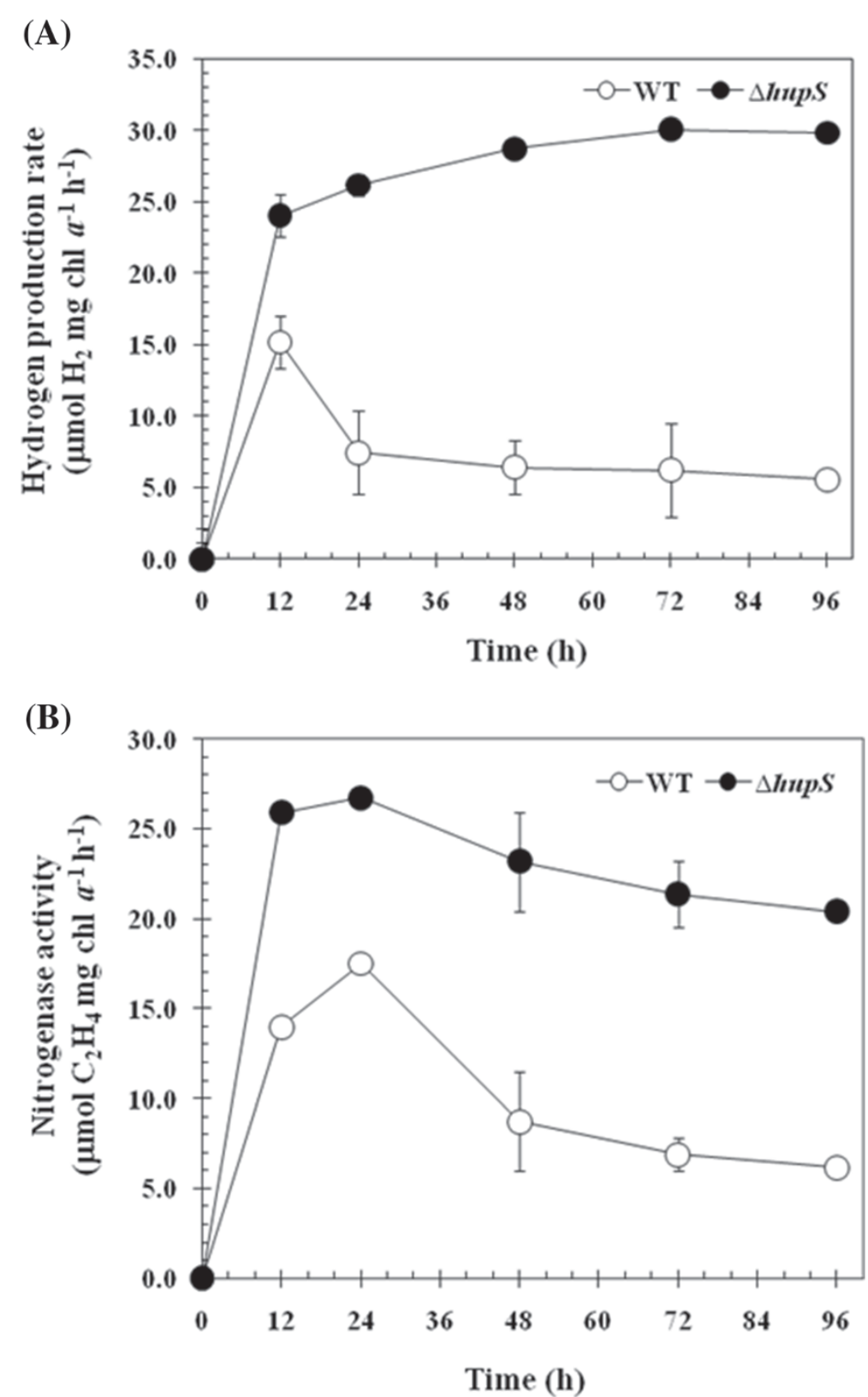

Figure 5 Effect of HupS inactivation on the sustainability of hydrogen production and nitrogenase activity. Comparison of hydrogen production (A) and nitrogenase activity (B) between wild type and $\Delta$ hupS strains of Anabaena siamensis TISTR 8012 was done after growth in BG110 medium for 4 days at $40 \mu \mathrm{Em}^{-2} \mathrm{~s}^{-1}$. The collected cells were determined for hydrogen production and nitrogenase activity after incubation for various times under continuous illumination of high light intensity at $200 \mu \mathrm{E} \mathrm{m} \mathrm{m}^{-2} \mathrm{~s}^{-1}$ and anaerobic condition. Means \pm S.D. $(n=3)$.

\section{Growth competition of wild type and $\Delta$ hupS strains in a mixed culture}

The ability of the $\Delta$ hupS strain of $A$. siamensis TISTR 8012 to compete with the wild type in a mixture without the addition of an antibiotic was studied using a molecular method to analyze the relative abundances of the two strains in a single sample. To demonstrate the possibility to use this method to quantify the relative abundance of the wild type and $\Delta$ hupS strains, axenic cultures of wild type and $\Delta$ hupS strains were mixed in known proportions before performing colony PCRs using primers specific to hupS. Obtained DNA fragments were analyzed by $0.8 \%$ agarose gel electrophoresis, their band intensities were quantified resulting in a standard calibration curve (Figure 7A). The method was then applied to an experiment analyzing the growth of the wild type and $\Delta$ hupS strains in a mixed culture for $120 \mathrm{~h}$. After around $50 \mathrm{~h}$ in the 50:50 mixed culture, the wild type started to grow on 

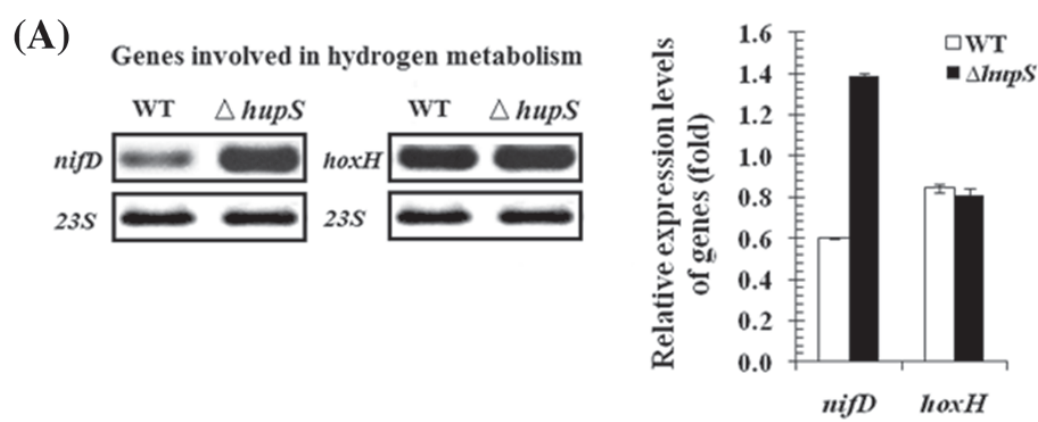

(B)

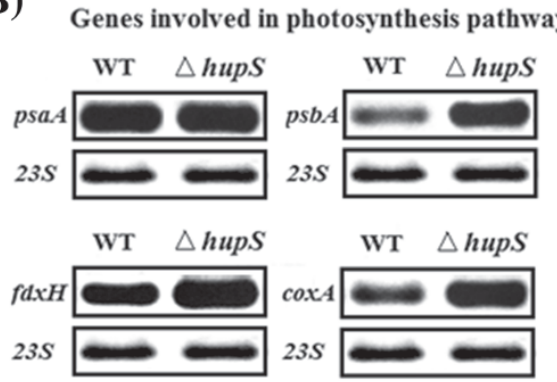

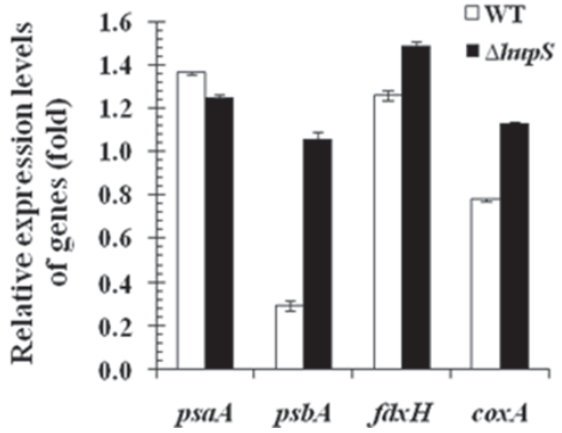

Figure 6 Transcript analyses of genes involved in hydrogen metabolism (A) and photosynthesis pathway (B). RT-PCR using total RNA isolated from wild type and $\triangle$ hupS cells grown in BG110 medium without $\mathrm{N}$-source for $12 \mathrm{~h}$. The PCR amplification using cDNAs of respective genes were performed using specific primers. 235 rRNA was used as control when determining the relative level of the respective transcript and the intensities of the PCR generated DNA fragments were determined by using GeneTools program.

the relative expense of the $\Delta$ hupS strain (Figure 7B), in agreement with an earlier observation using a $\triangle h u p L$ strain of Anabaena/Nostoc PCC 7120 [23]. The mixed culture of wild type and $\Delta$ hupS strains reached a ratio of 60:40 after $96 \mathrm{~h}$. It is worth noting that the $\Delta$ hupS strain may grow in antibiotic free medium without reverting back to wild type. However, the long term stability of the engineered strain should be further established in long term, large scale hydrogen production facilities.

\section{Conclusions}

This study further explores the potential for solar based biohydrogen production using a purpose designed engineered cyanobacterial strain, $\Delta$ hupS, using a general strategy outlined elsewhere [24]. We demonstrate that inactivation of hupS encoding the small subunit of the uptake hydrogenase in the cyanobacterium Anabaena siamensis TISTR 8012 results in long term sustainable, light dependent hydrogen production with enhanced nitrogenase activity and only minor effects on cell growth and heterocyst differentiation when compared with the wild type strain. The $\Delta$ hupS strain was found to compete well with the wild type up to $50 \mathrm{~h}$ in a mixed culture. However, the effects of HupS inactivation on general and specific metabolism as well as the long term stability of the $\Delta$ hupS strain warrant further investigations.

\section{Materials and methods}

\section{Strain and growth conditions}

The $\mathrm{N}_{2}$-fixing cyanobacterium Anabaena siamensis TISTR 8012 cells were grown in $50 \mathrm{~mL}$ of $\mathrm{BG}_{1} 1_{0}$ medium (without $\mathrm{N}$-source) and BG11 medium containing $18 \mathrm{mM}$ $\mathrm{NaNO}_{3}$ as $\mathrm{N}$-source [25], both media were buffered with $20 \mathrm{mM}$ HEPES- $\mathrm{NaOH}$ (pH 7.5). For $\Delta$ hupS strain, cells were grown in either $\mathrm{BG} 11$ or $\mathrm{BG} 11_{0}$ media containing $25 \mu \mathrm{g} \mathrm{mL}^{-1}$ neomycin antibiotic. The initial cell concentration was adjusted to an $\mathrm{OD}_{730}$ of 0.1 and cultures were incubated aerobically under continuous illumination of $40 \mu \mathrm{Em}^{-2} \mathrm{~s}^{-1}$ with cool white fluorescent lamps from two sides on a rotatory shaker at $160 \mathrm{rpm}$ and $30^{\circ} \mathrm{C}$. The growth rate was monitored by measuring the optical density of the culture at $730 \mathrm{~nm}$ with a spectrophotometer. The total amount of chlorophyll $a$ (chl $a$ ) was determined spectrophotometrically at $665 \mathrm{~nm}$ in $90 \%(\mathrm{v} / \mathrm{v})$ methanol extracts [26]. For the morphological study, A. siamensis TISTR 8012 cells were observed under Scanning Electron Microscope, SEM (JEOL model JSM-5410LV, Japan).

\section{Construct of recombinant plasmid and conjugative gene transfer}

The strategy for construction of recombinant plasmid containing target gene interruption could be divided into three steps as shown in Figure 1. The first step, the hupS gene sequence information in A. siamensis TISTR 8012 
(A)

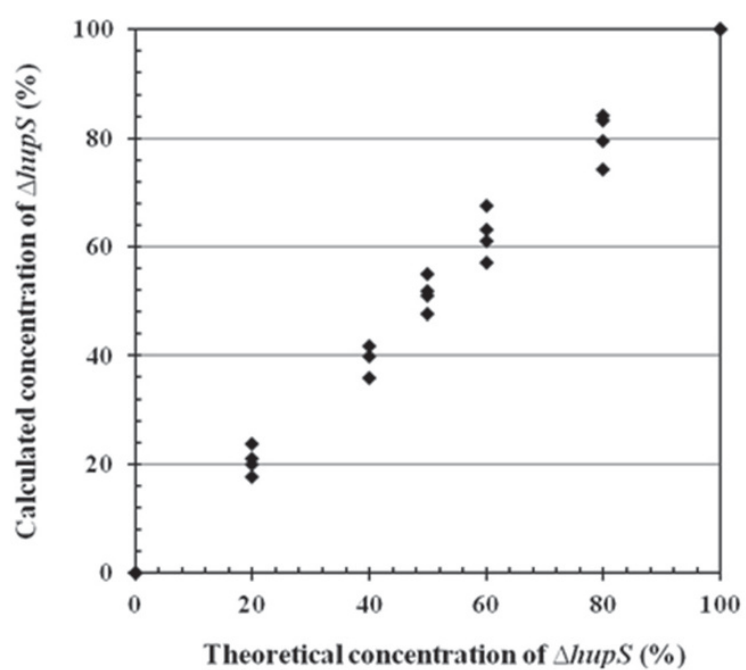

(B)

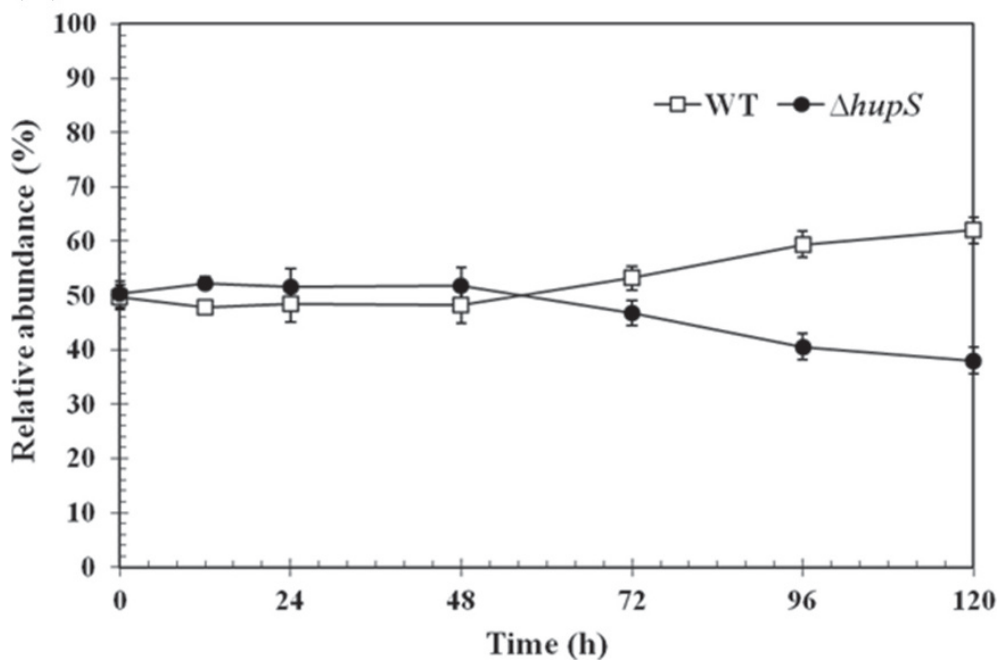

Figure 7 The ability of the $\Delta$ hupS strain to compete with the wild type strain analyzed using a molecular method to determine the relative abundances of the strains in mixed cultures. (A) Standard curve, theoretical and calculated \% of $\Delta$ hupS strain with respect to wild type in mixed cultures. (B) The relative abundance of wild type and $\Delta$ hupS strains. The relative abundance at time $=0$ was set at $50 \%$ (ratio of wild type to $\Delta$ hupS strain was 1:1).

was obtained from the NCBI database, accession number AY152844. hupS was amplified from extracted genomic DNA of A. siamensis TISTR 8012 cells by using specific primers, HupSF2 (gcatgcatgactaacgtactctggct) and HupSR2 (gcatgcgtctccattcccattaccta). The obtained hupS PCR product was purified and ligated into the pGEM-T easy vector (Promega), creating pGhupS plasmid. The second step, the $M l u I$ fragment containing a neomycin $(N m R)$ resistant cassette gene from pUC4K vector was modified blunt-ending and then inserted into EcoRV site within the hupS gene of the pGhupS plasmid to produce pGhupSNm plasmid. In the last step, a hupSNm fragment from pGhupSNm plasmid was amplified by using specific primers and then cloned into pRL271 vector to produce pRLhupSNm plasmid. pRLhupSNm plasmid functions as a cargo plasmid suitable to be transferred into A. siamensis TISTR 8012 cell. All plasmids were checked and confirmed by sequencing.

The cargo plasmid, pRLhupSNm was transformed into E. coli HB101 carrying the helper plasmid pRL623 and transferred to A. siamensis TISTR 8012 cell with the help of the conjugative plasmid pRL443 as shown in Table 1 by using the triparental mating method [27]. Single recombinant exconjugant colonies were selected on BG11 plate containing neomycin antibiotic at concentration of $25 \mu \mathrm{g} \mathrm{mL}{ }^{-1}$. To ensure the complete segregation, obtained gene knockout was analyzed by colony PCRs. 
Table 1 Plasmids used in this study

\begin{tabular}{lll}
\hline Plasmids & Relevant characteristic(s) & Source / Reference \\
\hline pGEM-T easy & Cloning vector, Apr lacZ', mcs & Promega \\
\hline pRL271 & $\begin{array}{l}\text { Cloning vector carrying sacB, } \\
\text { Em and Cm }\end{array}$ & $\begin{array}{l}\text { GenBank } \\
\text { accession \#L05081 }\end{array}$ \\
\hline pUC4K & Source of Nm cassette & Amersham \\
\hline pRL632 & $\begin{array}{l}\text { Helper plasmid carrying metylates } \\
\text { Aval, Avall and Avalll sites }\end{array}$ & {$[27]$} \\
\hline pRL443 & $\begin{array}{l}\text { Conjugative plasmid, Km } \\
\text { spontaneous mutant of RK2 }\end{array}$ & {$[27]$} \\
\hline pGhupS & $\begin{array}{l}\text { pGem-T easy vector contained } \\
\text { hupS }\end{array}$ & This study \\
\hline pGhupSNm & $\begin{array}{l}\text { Nm cassette inserted into EcoRV } \\
\text { site within hupS of pGhupS }\end{array}$ & This study \\
\hline pRLhupSNm & Cloning vector, Apr lacZ', mcs & This study \\
\hline
\end{tabular}

\section{Hydrogen production determination}

The cells were harvested and resuspended in $5 \mathrm{~mL}$ medium in a $13 \mathrm{~mL}$ of glass vial, then sealed with a rubber septum and a proper screw lid. The vial was bubbled with argon gas for 15 min to eliminate oxygen and incubated under different conditions at $30^{\circ} \mathrm{C}$ before determining hydrogen production. After $12 \mathrm{~h}$ incubation, a $400 \mu \mathrm{L}$ of head space gas sample was withdrawn from the vial with a gas tight syringe and the hydrogen gas was analyzed by a gas chromatograph (Peri-chrom PR2100, France) with a Molecular Sieve 5A 60/80 mesh column equipped with a thermal conductivity detector and argon as the carrier gas. The hydrogen production rate was expressed as $\mu \mathrm{mol} \mathrm{H}_{2} \mathrm{mg} \operatorname{chl} a^{-1} \mathrm{~h}^{-1}$.

\section{Nitrogenase activity determination}

In vivo nitrogenase activity was measured using the acetylene-reduction assay. In the absence of $\mathrm{N}_{2}$, the enzyme catalyzes the conversion of acetylene $\left(\mathrm{C}_{2} \mathrm{H}_{2}\right)$ to ethylene $\left(\mathrm{C}_{2} \mathrm{H}_{4}\right)$ gas. The reaction was carried out in a glass vial by incubation of the cells suspension $(2 \mathrm{~mL})$ with $1 \mathrm{~mL}$ of $10 \%(\mathrm{v} / \mathrm{v})$ acetylene $\left(\mathrm{C}_{2} \mathrm{H}_{2}\right)$ balanced in argon. The ethylene $\left(\mathrm{C}_{2} \mathrm{H}_{4}\right)$ production was detected by using a Gas Chromatograph with a Porapak Q, 50/80 mesh column equipped with a flame ionization detector (Shimadzu, Japan). Enzyme activity was expressed as $\mu \mathrm{mol} \mathrm{C} \mathrm{H}_{4} \mathrm{mg} \operatorname{chl} a^{-1} \mathrm{~h}^{-1}$.

\section{Transcription analysis}

The total RNA was extracted from cells in each condition by using the TRI Reagent ${ }^{\circledR}$ and treated with DNase (Fermentas) for DNA digestion. The treated RNA $(1 \mu \mathrm{g})$ was converted to single stranded cDNA with the iScript $^{\mathrm{TM}} \mathrm{cDNA}$ Synthesis Kit (Bio-RAD), according to the manufacturer's instruction. RT-PCR amplifications using cDNAs of the respective genes were performed using corresponding primers. Negative controls for the
RT-reaction were RT-PCR on DNaseI treated RNA without RT-enzyme. Negative controls for the PCR reactions were PCR amplification without $\mathrm{CDNA}$ added and positive controls were performed with genomic DNA using the corresponding primers. All primers used are listed in Table 2. The PCR conditions consisted of $95^{\circ} \mathrm{C}$ for $3 \mathrm{~min}$, followed by 30 cycles of $95^{\circ} \mathrm{C}$ for $15 \mathrm{sec}$, $50^{\circ} \mathrm{C}$ for $20 \mathrm{sec}$ and $72^{\circ} \mathrm{C}$ for $20 \mathrm{sec}$, and then a final extension at $72^{\circ} \mathrm{C}$ for $3 \mathrm{~min}$. The PCR product was analyzed by $1.0 \%(\mathrm{w} / \mathrm{v})$ agarose gel electrophoresis.

\section{Determination of the relative abundance of wild type and $\Delta$ hupS strain of Anabaena siamensis TISTR 8012 in a mixed culture}

Axenic cultures of $A$. siamensis TISTR 8012 wild type and the $\Delta$ hupS strain were mixed in known proportions. Colony PCRs were performed using primers specific to hupS and analyzed by $0.8 \%$ agarose gel electrophoresis. The sizes of the obtained PCR product were approximately $1.0 \mathrm{~kb}$ and $2.2 \mathrm{~kb}$ representing hupS of the wild type and hupS interrupted with neomycin resistant cassette gene of the engineered strain, respectively. The intensities of the PCR bands were compared within each lane and calculated by using GeneTools program (SynGene, USA) to detect the presence and the relative abundance of the wild type and $\Delta$ hupS strain in a single sample. Using this protocol a standard curve was generated and shown as percent of $\Delta h u p S$ with respect to wild type strain. For competition experiments, the axenic cultures of wild type and $\Delta$ hupS strains of A. siamensis

Table 2 Primers used in RT-PCR reactions

\begin{tabular}{llll}
\hline Primer & Sequence $\mathbf{5}^{\prime}$ to $\mathbf{3}$ & $\begin{array}{l}\text { Target of } \\
\text { primer pair }\end{array}$ & $\begin{array}{l}\text { PCR } \\
\text { product, bp }\end{array}$ \\
\hline ASnifDF1 & tcgtattcggtggtgacaaa & nifD & 204 \\
\hline ASnifDR1 & gagacacaccacggaaacct & & \\
\hline AShoxHF1 & gaatccgtctgcgtcaattt & hoxH & 284 \\
\hline AShoxHR1 & gcaaatgtccgtcgtaggtt & & \\
\hline 23F & gctaagcgatgtaccgaagc & 23S rDNA & 200 \\
\hline 23R & taacccagagtggacgaacc & & \\
\hline PsaAF1 & ctgttgaaaggtgtattgtt & psaA & 489 \\
\hline PsaAR1 & aggagctaccttcagtttat & & 390 \\
\hline PsbAF1 & gcacattcaactttatgatt & psbA & \\
\hline PsbAR1 & ccaaaattgagttattgaag & & 321 \\
\hline FdxHF1 & atggctagctaccaagttag & fdxH & \\
\hline FdxHR1 & ttaagcaaggtacggttctt & & 299 \\
\hline CoxAF1 & gcgagattacttcagttta & coxA & \\
\hline CoxAR1 & atccaaataccttctcctac & & \\
\hline NtcAF1 & cgagtctactttctttgaa & $n t c A$ & \\
\hline NtcAR1 & aaaatcacgacagagaatta & & \\
\hline HetRF & ggatgaccggacattgcac & hetR & \\
\hline HetRR & ccataagcgatcgcaagagg & & \\
\hline & & & \\
\hline
\end{tabular}


TISTR 8012 were mixed together in a $50 \mathrm{~mL}$ growth flask in a ratio of $1: 1$ based on $\mathrm{OD}_{730}$ measurements and incubated under growth conditions. The relative abundance of the wild type versus the $\Delta$ hupS strain was then calculated during the experiment.

\section{Competing interest}

The authors declare that they have no competing interests.

\section{Authors' contributions}

WK, PL and Al designed the study, analyzed the data and wrote the manuscript. WK performed the experiments. All authors read and approved the final manuscript

\section{Acknowledgements}

This work was supported by the Royal Golden Jubilee Ph.D. program (PHD/0147/2549), the $90^{\text {th }}$ Anniversary of Chulalongkorn University Fund (Ratchadaphiseksomphote Endowment Fund) to W. Khetkorn and A. Incharoensakdi, the Swedish Energy Agency, and the Swedish Research Links program (project 348-2009-6486). The research grants by Commission on Higher Education, Thailand (CHE) (the university staff development consortium), the Thai government SP2 (TKK2555) and the National Research University Project, CHE (FW0659A) to A. Incharoensakdi is also acknowledged. W. Khetkorn also thanks the Graduate School of Chulalongkorn University for providing post-doctoral Fellowship.

Received: 14 June 2012 Accepted: 7 October 2012

Published: 10 October 2012

\section{References}

1. Khetkorn W, Lindblad P, Incharoensakdi A: Enhanced biohydrogen production by the $\mathrm{N}_{2}$-fixing cyanobacterium Anabaena siamensis strain TISTR 8012. Int J Hydrogen Energy 2010, 35:12767-12776.

2. Tamagnini P, Leitao E, Oliveira P, Ferreira D, Pinto F, Harris DJ, Heidorn T, Lindblad P: Cyanobacterial hydrogenase: diversity, regulation and applications. FEMS Microbiol Rev 2007, 31:692-720.

3. Tamagnini $P$, Axelsson $R$, Lindberg P, Oxelfelt F, Wunschiers R, Lindblad P: Hydrogenase and hydrogen metabolism of cyanobacteria. Microbiol Mol Rev 2002, 66:1-20.

4. Houchins JP, Burris RH: Light and dark reactions of the uptake hydrogenase in Anabaena 7120. Plant Physiol 1981, 68:712-716.

5. Lindblad $P$, Sellstedt $A$ : Occurrence and localization of an uptake hydrogenase in the filamentous heterocystous cyanobacterium Nostoc PCC 73102. Protoplasma 1990, 159:9-15.

6. Kentemich T, Casper M, Bothe H: The reversible hydrogenase in Anacystis nidulans is a component of the cytoplasmic membrane. Naturwissenschaften 1991, 78:559-560.

7. Khetkorn W, Baebprasert W, Lindblad P, Incharoensakdi A: Redirecting the electron flow towards the nitrogenase and bidirectional Hoxhydrogenase by using specific inhibitors results in enhanced $\mathrm{H}_{2}$ production in the cyanobacterium Anabaena siamensis TISTR 8012. Bioresource Technol 2012, 118:265-271.

8. Happe T, Schutz K, Bohme H: Transcriptional and mutational analysis of the uptake hydrogenase of the filamentous cyanobacterium Anabaena variabilis ATCC 29413. J Bacteriol 2000, 182:1624-1631.

9. Lindberg P, Schutz K, Happe T, Lindblad P: A hydrogen-producing, hydrogenase-free mutant strain of Nostoc punctiforme ATCC 29133. Int J Hydrogen Energy 2002, 27:1291-1296.

10. Masukawa H, Mochimaru M, Sakurai H: Disruption of the uptake hydrogenase gene, but not of the bi-directional hydrogenase gene, leads to enhanced photobiological hydrogen production by the nitrogen-fixing cyanobacterium Anabaena sp. PCC 7120. Appl Microbiol Biotechnol 2002, 58:618-624.

11. Carrasco CD, Holliday SD, Hansel A, Lindblad P, Golden JW: Heterocyst-specific excision of the Anabaena sp. strain PCC 7120 hupL element requires xisC. J Bacteriol 2005, 187:6031-6038.

12. Yoshino F, Ikeda H, Masukawa H, Sakurai H: High photobiological hydrogen production activity of a Nostoc sp. PCC 7422 uptake hydrogenase-deficient mutant with high nitrogenase activity. Mar Biotechnol 2007, 9:101-112.
13. Phunpruch S, Baebprasert W, Thongpeng C, Incharoensakdi A: Nucleotide sequencing and transcriptional analysis of uptake hydrogenase genes in the filamentous $\mathrm{N}_{2}$-fixing cyanobacterium Anabaena siamensis. J Appl Phycol 2006, 18:713-722.

14. Bock A, King PW, Blokesch M, Posewitz MC: Maturation of hydrogenases. Adv Microb Physiol 2006, 51:1-71.

15. Frias JE, Flores $E$, Herrero A: Requirement of the regulatory protein NtcA for the expression of nitrogen assimilation and heterocyst development genes in the cyanobacterium Anabaena sp. PCC 7120. Mol Microbiol 1994, 14:823-832

16. Wei TF, Ramasubramanian TS, Golden JW: Anabaena sp. strain PCC 7120 $n t c A$ gene required for growth on nitrate and heterocyst development. J Bacteriol 1994, 176:4473-4482.

17. Huang $X$, Dong $Y$, Zhao J: HetR homodimer is a DNA-binding protein required for heterocyst differentiation, and the DNA-binding activity is inhibited by PatS. Proc Natl Acad Sci USA 2004, 2004(101):4848-4853.

18. Buikema WJ, Haselkorn R: Expression of the Anabaena hetR gene from a copper-regulated promoter leads to heterocyst differentiation under repressing condition. Proc Natl Acad Sci USA 2001, 98:2729-2734.

19. Black TA, Cai YP, Wolk CP: Spatial expression and autoregulation of hetR, a gene involved in the control of heterocyst development in Anabaena. Mol Microbiol 1993, 9:77-84.

20. Muro-Pastor AM, Valladares A, Flores E, Herrero A: Mutual dependence of the expression of the cell differentiation regulatory protein HetR and the global nitrogen regulator NtcA during heterocyst development. $\mathrm{Mol}$ Microbiol 2002, 44:1377-1385.

21. Ekman M, OW SY, Holmqvist M, Zhang X, Wangenen JV, Wright PC, Stensjö $\mathrm{K}$ : Metabolic adaptations in a $\mathrm{H}_{2}$ producing heterocyst-forming cyanobacterium: potentials and implications for biological engineering. J Proteome Res 2011, 10:1772-1784

22. Valladares A, Herrero A, Pils D, Schmetterer G, Flores E: Cytochrome c oxidase genes required for nitrogenase activity and diazotrophic growth in Anabaena sp. PCC 7120. Mol Microbiol 2003, 47:1239-1249.

23. Lindblad $P$, Christensson $K$, Lindberg $P$, Pinto $F$, Tsygankov A: Photoproduction of $\mathrm{H}_{2}$ by wildtype Anabaena PCC 7120 and a hydrogen uptake deficient mutant: from laboratory experiments to outdoor culture. Int J Hydrogen Energy 2002, 27:1271-1281.

24. Lindblad P, Lindberg P, Oliveira P, Stensjö K, Heidorn T: Design, engineering, and construction of photosynthetic microbial cell factories for renewable solar fuel production. Ambio 2012, 41(Suppl 2):163-168.

25. Rippka R, Deruelles J, Waterbury JB, Herdman M, Stanier RY: Generic assignments, strain histories and properties of pure cultures of cyanobacteria. J Gen Microbiol 1979, 111:1-61.

26. Mackinney G: Absorption of light by chlorophyll solutions. J Biol Chem 1941, 140:315-322.

27. Elhai J, Wolk CP: Conjugal transfer of DNA to cyanobacteria. Methods Enzymol 1988, 167:747-754.

doi:10.1186/1754-1611-6-19

Cite this article as: Khetkorn et al: Inactivation of uptake hydrogenase leads to enhanced and sustained hydrogen production with high nitrogenase activity under high light exposure in the cyanobacterium Anabaena siamensis TISTR 8012. Journal of Biological Engineering 2012 6:19.

\section{Submit your next manuscript to BioMed Central and take full advantage of:}

- Convenient online submission

- Thorough peer review

- No space constraints or color figure charges

- Immediate publication on acceptance

- Inclusion in PubMed, CAS, Scopus and Google Scholar

- Research which is freely available for redistribution 